\title{
A universality of dark-halo surface density for the Milky Way and Andromeda dwarf satellites as a probe of the coldness of dark matter
}

\author{
Kohei Hayashi ${ }^{1}$ and Masashi Chiba ${ }^{2}$ \\ ${ }^{1}$ Kavli Institute for the Physics and Mathematics of the Universe, University of Tokyo, \\ 5-1-5 Kashiwa-no-ha, Kashiwa, 277-8568, Japan \\ email: kohei.hayashi@ipmu.jp \\ ${ }^{2}$ Astronomical Institute, Tohoku University, \\ 6-3 Aoba-ku, Sendai, 980-8578, Japan \\ email: chiba@astr.tohoku.ac.jp
}

\begin{abstract}
We propose a new astrophysical test on the nature of dark matter based on the properties of dark halos associated with dwarf spheroidal galaxies. The method adopts a mean surface density of a dark halo defined within a radius of maximum circular velocity, which is derivable for a wide variety of galaxies with any dark-matter density profiles. We find that even though dark halo density profiles are derived based on the different assumptions for each galaxy sample, this surface density is generally constant across a wide mass range of galaxy. We find that at higher halo-mass scales, this constancy for real galaxies can be naturally reproduced by both cold and warm dark matter (CDM and WDM) models. However, at low-mass scales, for which we have estimated from the Milky Way and Andromeda dwarf satellites, the mean surface density derived from WDM models largely deviates from the observed constancy, whereas CDM models are in reasonable agreement with observations.
\end{abstract}

\section{Mean Surface Density of Dark Halo}

In order to obtain severer limits on dark matter models, we define the mean surface density of a dark matter halo within the radius of maximum circular velocity,

$$
\Sigma_{\mathrm{V}_{\max }}=\frac{M\left(r_{\max }\right)}{\pi r_{\max }^{2}},
$$

where

$$
M\left(r_{\max }\right)=\int_{0}^{r_{\max }} 4 \pi \rho_{\mathrm{dm}}\left(r^{\prime}\right) r^{\prime 2} d r^{\prime}
$$

$\rho_{\mathrm{dm}}(r)$ indicates any dark matter density profiles, and $r_{\mathrm{max}}$ is a radius at maximum circular velocity, $V_{\max }$, of assumed dark halo profiles, $\rho_{\mathrm{dm}}$.

Using this definition (1.1), we estimate $\Sigma_{\mathrm{V}_{\max }}$ for late- and early-type spirals with pseudo-isothermal dark halos (de Blok et al. 2008; Spano et al. 2008), dwarf irregulars with Burkert dark halos (Gentile et al. 2005, 2007), galaxy-galaxy weak lensing sample of spiral and elliptical galaxies with Burkert profiles (Donato et al. 2009), and MW's and M31's dwarf spheroidal galaxies with double-power law density profiles (Hayashi \& Chiba 2015). The symbols with error bars in Figure 1 show the estimated mean surface density, $\Sigma_{V_{\max }}$, of the above data sample as a function of $V_{\max }$. It is found that even though dark halo density profiles are derived based on the different assumptions for each 


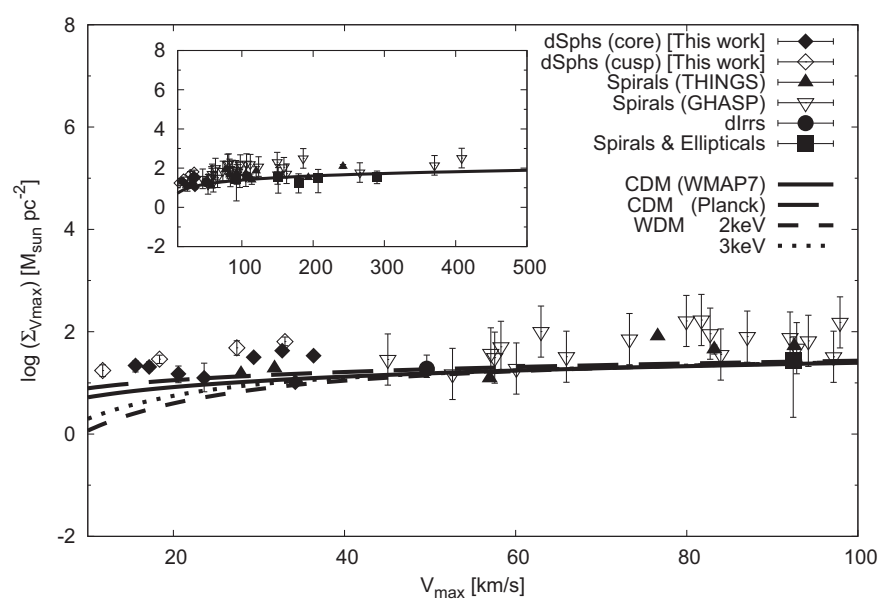

Figure 1. Mean surface density of a dark halo, $\Sigma_{V_{\max }}$ as a function of $V_{\max }$ for different type of galaxies (each point with error bars) and for theoretical prediction from $\Lambda$ CDM (solid lines) and $\Lambda$ WDM-based (dashed lines) $N$-body simulations.

galaxy sample and estimated by several independent methods, this surface density is sufficiently constant across a wide range of $V_{\max }$ of about 10 to $400 \mathrm{~km} \mathrm{~s}^{-1}$.

\section{Constraints on dark matter models}

This mean surface density can be directly computed from the theoretical predictions based on cosmological $N$-body simulations within the $\Lambda$-dominated CDM and WDM models. First, we adopt the NFW dark matter density profile, which can reproduce CDM (Navarro et al. 1997) and WDM (Lovell et al. 2014) dark halos. We then evaluate the scale density and the scale length in the cosmological context and the mass-concentration relation at $z=0$. Finally, using dark matter density profiles obtained by the above procedure, we calculate $\Sigma_{V_{\max }}$ for CDM (WMAP7 and Planck) and WDM (particle masses with 2 and $3 \mathrm{keV}$ ) models.

The solid and dashed lines in Figure 1 indicate the predicted $\Sigma_{V_{\max }}$ versus $V_{\max }$ for CDM and WDM models, respectively. It is found that at higher halo-mass scales, this constancy for real galaxies can be naturally reproduced by both dark matter models, even though we do not perform any fitting to the data. However, at dwarf-galaxy mass scales the mean surface density derived from WDM models deviates systematically from the observed universality, whereas CDM models are in reasonable agreement with observations. Thus, this test supports CDM models.

\section{References}

de Blok, W. J. G., Walter, F., Brinks, E., et al. 2008, AJ, 136, 2648

Donato, F., Gentile, G., Salucci, P., et al. 2009, MNRAS, 397, 1169

Gentile, G., Burkert, A., Salucci, P., Klein, U., \& Walter, F. 2005, ApJL, 634, L145

Gentile, G., Salucci, P., Klein, U., \& Granato, G. L. 2007, MNRAS, 375, 199

Hayashi, K. \& Chiba, M. 2015, ApJ, 810, 22

Lovell, M. R., Frenk, C. S., Eke, V. R., et al. 2014, MNRAS, 439, 300

Navarro, J. F., Frenk, C. S., \& White, S. D. M. 1997, ApJ, 490, 493

Spano, M., Marcelin, M., Amram, P., et al. 2008, MNRAS, 383, 297 